\title{
A RESULT RELATED TO A THEOREM BY PIANIGIANI
}

\author{
ABRAHAM BOYARSKY ${ }^{1}$ AND GABRIEL HADDAD
}

\begin{abstract}
ABSTRACr. Let $\tau: J \rightarrow J$ be a piecewise $C^{2}$ map, where $J$ is an interval, satisfying inf $\left|\tau^{\prime}\right|>1$. An upper bound for the number of independent absolutely continuous measures invariant under $\tau$ is presented.
\end{abstract}

Introduction. Let $J=[a, b]$ be an interval, $\mathscr{B}$ the Lebesgue measurable subsets of $J$, and $\lambda$ the Lebesgue measure on $J$. Let $\tau: J \rightarrow J$ be a piecewise $C^{2}$ transformation satisfying $\inf \left|\tau^{\prime}(x)\right|>1$ where the derivative exists. In [1] it is shown that $\tau$ admits an absolutely continuous invariant measure $\mu$, i.e., $\mu(A)=$ $\mu\left(\tau^{-1}(A)\right)$ for all $A \in \mathscr{B}$, and

$$
\mu(A)=\int_{A} f d \lambda
$$

where we refer to $f$ as the density invariant under $\tau$. Clearly $f>0$ and $f \in \mathcal{L}_{1}$, the space of integrable functions on $J$.

Let $\mathscr{F}_{\tau}$ denote the space of densities invariant under $\tau$ and $\left\{a_{1}, a_{2}, \ldots, a_{k}\right\}$ those points in $J$ where $\tau^{\prime}$ does not exist. The main result of [2] asserts that $\operatorname{dim} \mathscr{F}_{\tau}<k$. In fact it is very easy to establish a better bound. Let $a=b_{0}<b_{1}<\cdots<b_{m}<$ $b_{m+1}=b$ be the partition of $J$ such that $\tau$ is continuous and monotonic on each interval $\left(b_{i-1}, b_{i}\right)$. Clearly $m \leqslant k$, and $\operatorname{dim} \mathscr{F}_{\tau} \leqslant m$. In the special case where $\tau$ is continuous on $J$, the total number of peaks and valleys in the graph of $\tau$ constitutes an upper bound for $\operatorname{dim} \mathscr{F}_{\tau}$.

In $\$ 3$ of [3] a still better bound is established for $\operatorname{dim} \mathscr{F}_{\tau}$. Let $\left\{b_{1}, b_{2}, \ldots, b_{m}\right\}$ be the partition defined in the previous paragraph. For each $1<j<m$, define the pair

$$
\left\langle u_{j}, v_{j}\right\rangle=\left\langle\tau\left(b_{j}^{-}\right), \tau\left(b_{j}^{+}\right)\right\rangle
$$

where $u_{j}$ is regarded as $u_{j}^{+}$or $u_{j}^{-}$depending on whether $\tau\left(a_{j}-\varepsilon\right)>u_{j}$ or $\tau\left(a_{j}-\varepsilon\right)$ $<u_{j}$.

Two pairs $\left\langle u_{i}, v_{i}\right\rangle$ and $\left\langle u_{j}, v_{j}\right\rangle$ are said to be dependent if they have one or both coordinates in common. Otherwise the pairs are independent. Let $N_{\tau}$ denote the maximal number of independent pairs. Then Theorem 2 of [3] asserts that $\operatorname{dim} \mathscr{F}_{\tau}$ $<N_{\tau}$. In this note we suggest a modified definition of dependence and present a different bound for the number of absolutely continuous measures invariant under $\tau$.

Received by the editors October 15, 1980.

1980 Mathematics Subject Classification. Primary 26A18; Secondary 28D05.

1 The research of this author was supported by NSERC Grant \# A-9072.

(c) 1981 American Mathematical Society 0002-9939/81/0000-0356/\$01.75 
2. Dependence of densities. Let $\tau: J \rightarrow J$ be piecewise $C^{2}$ satisfying inf $\left|\tau^{\prime}(x)\right|>1$ and let $\mathscr{D}=\left\{b_{1}, b_{2}, \ldots, b_{m}\right\}$ be the partition on which $\tau$ is piecewise continuous and monotonic. We shall say that $b_{i}$ and $b_{j}$ are dependent if

$$
\tau\left(b_{i}-\varepsilon, b_{i}+\varepsilon\right) \cap \tau\left(b_{j}-\varepsilon, b_{j}+\varepsilon\right)
$$

has positive measure for every $\varepsilon>0$. This implies, but is not equivalent to

$$
\left\langle\tau\left(b_{i}^{-}\right), \tau\left(b_{i}^{+}\right)\right\rangle \cap\left\langle\tau\left(b_{j}^{-}\right), \tau\left(b_{j}^{+}\right)\right\rangle \neq \varnothing .
$$

This definition of dependence for a pair of discontinuities in $\mathscr{D}$ is reflexive, symmetric, but not transitive. A collection $\mathcal{S} \subset \mathscr{D}$ is said to be dependent if every pair of points in this collection is dependent, and maximal if $\delta$ is not a proper subset of any dependent collection. Notice that two distinct maximal dependent collections may have nonempty intersection, and such a collection may consist of a single point. Thus, given $b_{j} \in \mathscr{D}$, there exists at least one and at most two maximal dependent collections containing $b_{j}$. In particular, when $\tau$ is continuous at $b_{j}$, there exists only one maximal dependent collection containing this point. Let $H_{\tau}$ be the number of distinct maximal dependent collections. Then, we have

THEOREM. $\operatorname{dim} \mathscr{F}_{\tau} \leqslant H_{\tau}$.

Proof. We first show that if $f_{1}$ and $f_{2}$ are invariant with disjoint supports, then to each $f_{i}$ there corresponds one maximal dependent collection $S_{i}$ and $S_{1} \neq S_{2}$. Letting $M_{i}=\operatorname{spt} f_{i}$, it is easy to see that int $M_{i}$ has to contain at least one point of $\mathscr{D}$, say $b_{i}^{\prime}$. Let $S_{1}$ and $S_{2}$ be any maximal collections containing $b_{1}^{\prime}$ and $b_{2}^{\prime}$, respectively, and suppose $S_{1}=S_{2}$. Then $b_{1}^{\prime}$ and $b_{2}^{\prime}$ are dependent. Since $\tau\left(M_{i}\right) \subset$ $M_{i}$ a.e. [1], and $\left(b_{i}^{\prime}-\varepsilon, b_{i}^{\prime}+\varepsilon\right) \subset M_{i}$ for some $\varepsilon<0$, the dependence of $b_{1}^{\prime}$ and $b_{2}^{\prime}$ implies

$$
\lambda\left(M_{1} \cap M_{2}\right) \geqslant \lambda\left[\tau\left(b_{1}^{\prime}-\varepsilon, b_{1}^{\prime}+\varepsilon\right) \cap \tau\left(b_{2}^{\prime}-\varepsilon, b_{2}^{\prime}+\varepsilon\right)\right]>0 .
$$

This is a contradiction. Therefore, $S_{1}$ and $S_{2}$ must be distinct.

Now let $\left\{f_{1}, f_{2}, \ldots, f_{n}\right\}$ be a maximal set of disjoint densities invariant under $\tau$ [2]. By the preceding argument we see that there exists a 1-1 mapping from $\left\{f_{1}, \ldots, f_{n}\right\}$ into $\left\{S_{1}, \ldots, S_{H_{\tau}}\right\}$. Thus $n \leqslant H_{\tau}$. Q.E.D.

3. Examples. (a) Consider the transformation $\tau$ shown in Figure 1.

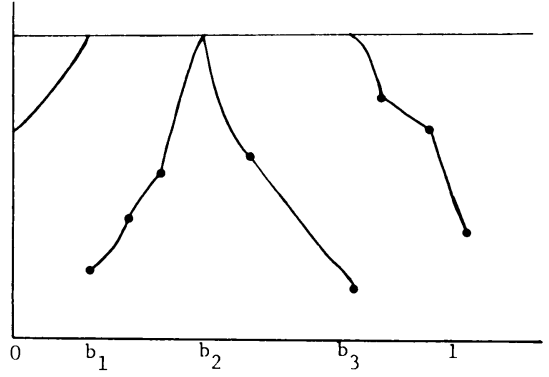

FIGURE 1

We see that $\left\{b_{1}, b_{2}, b_{3}\right\}$ is the unique collection which is dependent and maximal. Thus $H_{\tau}=1$ and there exists a unique absolutely continuous measure invariant under $\tau$. The bound from [2] is 8 , since there are 8 discontinuities in $\tau^{\prime}$ in $(0,1)$. 
(b) Let $\tau$ have the graph shown in Figure 2.

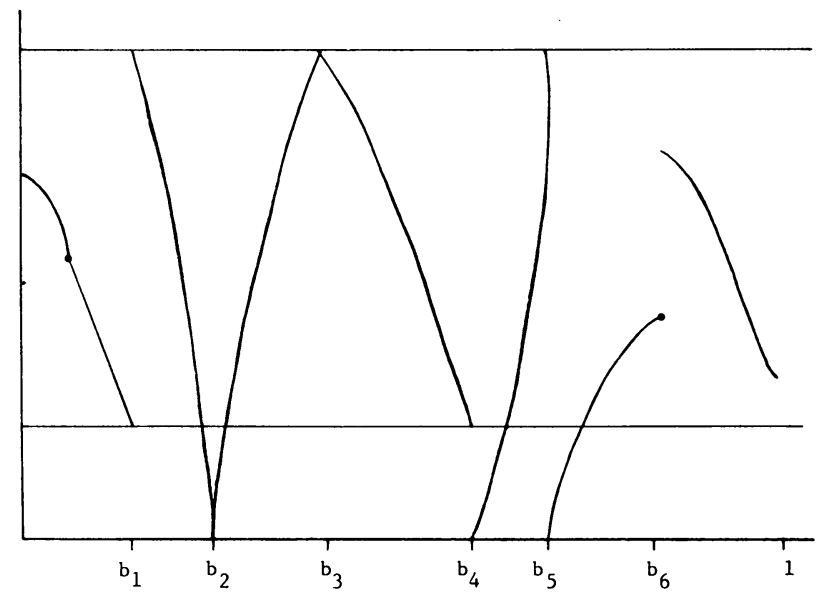

FIGURE 2

For each discontinuity, we give the corresponding maximal dependent collection or collections as the case may be:

$b_{1}:\left\{b_{1}, b_{3}, b_{5}\right\}$ and $\left\{b_{1}, b_{4}\right\}$,

$b_{2}:\left\{b_{2}, b_{4}, b_{5}\right\}$,

$b_{3}:\left\{b_{1}, b_{3}, b_{5}\right\}$,

$b_{4}:\left\{b_{1}, b_{4}\right\}$ and $\left\{b_{2}, b_{4}, b_{5}\right\}$,

$b_{5}:\left\{b_{1}, b_{3}, b_{5}\right\}$ and $\left\{b_{2}, b_{4}, b_{5}\right\}$,

$b_{6}:\left\{b_{6}\right\}$.

There are 4 independent collections. Therefore $\tau$ admits at most four independent invariant densities.

Notice that for this example the bound of [2] is 7 , since there are 7 discontinuities of $\tau^{\prime}$ in $(0,1)$.

\section{REFERENCES}

1. A. Lasota and J. A. Yorke, On the existence of invariant measures for piecewise monotonic transformations, Trans. Amer. Math. Soc. 186 (1973), 481-486.

2. T. Y. Li and J. A. Yorke, Ergodic transformations from an interval into itself, Trans. Amer. Math. Soc. 235 (1978), 183-192.

3. G. Pianigiani, First return map and invariant measures, Israel J. Math. 35 (1980), $32-48$.

Department of Mathematics, Sir George Williams Campus, Concordin Universtty, Montreal, Quebec, Canada H3G $1 \mathrm{M} 8$ 